\title{
PRESSURE DEPENDENCE OF THE SCHOTTKY BARRIER HEIGHTS IN Al/AlGaAs JUNCTIONS*
}

\author{
L. Dobaczewski, J.M. Langer \\ Institute of Physics, Polish Academy of Sciences \\ Al. Lotników 32/46, 02-668 Warszawa, Poland
}

AND M. Missous

University of Manchester, Institute of Science and Technology

P.O. Box 88, Manchester M60 1QD, The United Kingdom

The influence of hydrostatic pressure up to $8 \mathrm{kbar}$ on the barrier height of epitaxially MBE-grown $\mathrm{Al}$ on AlGaAs metal-semiconductor junctions is reported. The pressure change of the Schottky barrier on $n$-type AlGaAs is the same as that of the energy gap (for both direct and indirect-gap AlGaAs compositions), while for $p$-type AlGaAs it is negligible. This result is in direct conflict with a class of models of the Schottky barrier formation based on a concept of a semiconductor neutrality level alignment with the metal Fermi level.

PACS numbers: $73.30 .+\mathrm{y}, 73.40 . \mathrm{Kp}, 73.20 . \mathrm{Hb}$

It is well established that the barrier height of metal-semiconductor (MS) contacts made on covalent semiconductors are almost independent on the metal work function. Such a behaviour indicates that some generic mechanism must be responsible for the Fermi level pinning. One group of models stresses the role of the metal-induced gap states (MIGS) [1, 2]. These states are defined by a band structure of a semiconductor and a pinning energy corresponds to the neutrality level of a semiconductor. This elegant idea ignores completely all chemical reactions occurring at the interface during the formation of a contact. It is known that surface atoms undergo rebonding. Also, metal deposition may cause generation of defects during metallization [3-5]. Such defects may also cause the Fermi level pinning. Each of these models, i.e. MIGS or defect model has certain merits. It may well be that in reality it is a combined action of both mechanisms that causes the pinning. It has recently been proposed that studying of the influence of external perturbation (such as temperature or hydrostatic pressure) upon the barrier height may show which of the two mechanisms is more important [6].

The neutrality level reflects an average of the whole band structure, therefore it should be insensitive in the first order to external perturbations. Hence the

\footnotetext{
* This work is supported in part by the grant No. 880979102 of the Committee for Scientific Research in Poland and by the SERC grants in UK.
} 
variation of the Schottky barrier height on $n$-type and $p$-type semiconductor should follow the individual shift of the conduction and the valence band, respectively. On the other hand, if defects caused the Fermi level pinning, the change of the barrier would reflect the motion of the defect relative to the appropriate band edge.

Accurate measurements of the temperature evolution of the Schottky barriers in epitaxially grown $\mathrm{Al} / \mathrm{AlGaAs} \mathrm{MS}$ junctions have been done recently [6-8]. We found that the temperature dependence of the Al/AlGaAs ( $n$-type) follows the temperature dependence of the energy gap (for all AlGaAs compositions, i.e., in both direct and indirect gap composition range). The temperature dependence of the barrier height on the $p$-type material was much weaker, if any.

These results provided the first direct indication that the concept of the charge neutrality level may not be valid in case of the MBE-grown metal-semiconductor junctions made on AlGaAs and were interpreted as consistent with a defect model of the Schottky barrier formation, provided the defects causing the Fermi level pinning were localised and of a bonding type.

An alternative way of testing validity of the neutrality level concept is to measure an isothermal pressure dependence of the barrier height. If the MIGS was dominated by the barrier formation, then the influence of pressure would have been given by the deformation potentials $a_{\mathrm{cb}}$ or $a_{\mathrm{vb}}$ for the barriers on $n$ - and $p$-type substrates, respectively. Although these quantities are hardly accessible from direct measurements, there is a certain consensus as to their signs and values [9]. For most direct-band gap materials $\left|a_{\mathrm{vb}}\right| \ll\left|a_{\mathrm{cb}}\right|$ and thus the pressure experiment may be not conclusive. However, for the indirect-band gap materials the values of the deformation potentials $a_{\mathrm{vb}}$ and $a_{\mathrm{cb}}$ are very close and their signs are the same [9].

The Al/AlGaAs junctions measured in this work were the same as used previously in determining the temperature evolution of the Schottky barrier heights. They were grown by the MBE technique in one growth cycle (see Ref. [10] for details), and thus all layers (AlGaAs and Al-metallization) were of epitaxial quality. Pressure measurements were done in UNIPRESS piston-type transport high pressure cells at room temperature. As the $I(V)$ characteristics of the junctions were almost ideal (the ideality factor $\eta$ in $\mathrm{Eq}$. (1) was close to unity), this type of measurement was chosen for the determination of the change of the Schottky barrier height with pressure.

The current density-voltage $I(V)$ characteristics of an MS junction is given by a relation

$$
I=I_{0} \exp (q V / \eta k T)[1-\exp (-q V / k T)],
$$

where $\eta$, known as the ideality factor, is used as a guide in determining the quality of the diodes. If the value of $\eta$ is close to unity, then the conditions of a pure thermionic theory are fulfilled, and the $I(V)$ characteristics can be used in the derivation of the barrier height.

At elevated temperatures (i.e. well above the tunnelling regime) and for moderate doping the saturation current density $I_{0}$ is given by

$$
I_{0}=A^{*} T^{2} \exp \left(-q \Phi_{\mathrm{b}} / k T\right),
$$

where $\Phi_{\mathrm{b}}$ is the barrier height corrected for the image force barrier lowering and $A^{*}$ is the effective Richardson constant. Therefore, under isothermal conditions the 
pressure change of the barrier height is just a change under pressure of $\ln \left(I_{0}\right)$. Small corrections may be necessary if the effective mass entering $A^{*}$ changes substantially with the pressure.
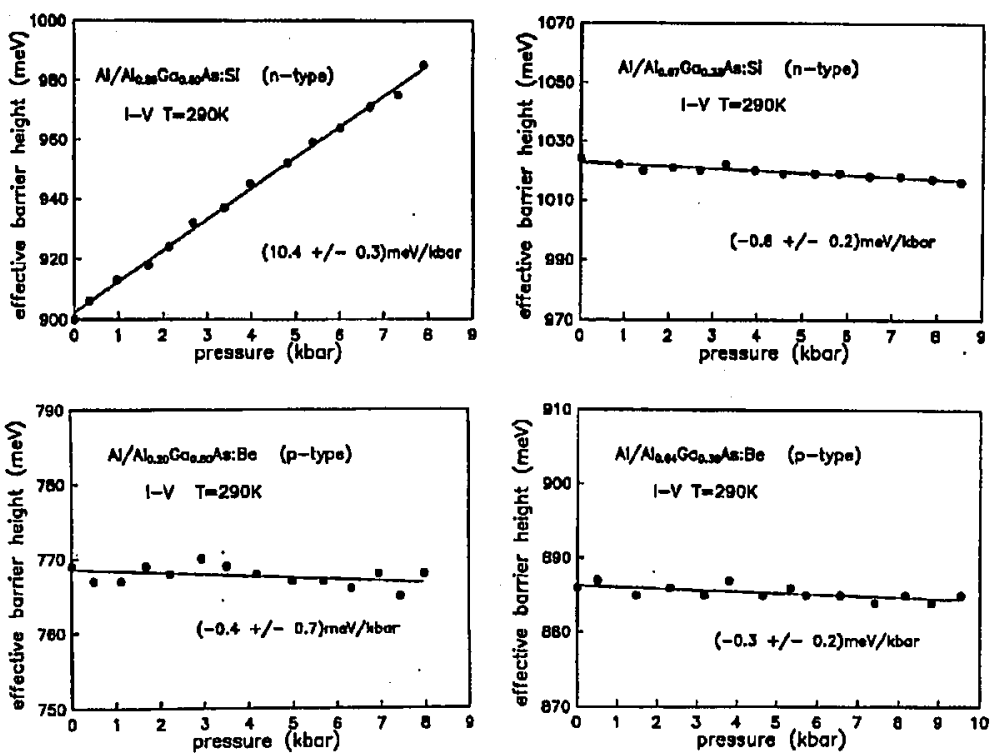

Fig. 1. Pressure dependence of the effective Schottky barrier heights on $n$ - and $p$-type $\mathrm{Al} / \mathrm{AlGaAs}$ junctions for direct and indirect gap AlGaAs compositions.

The results of the pressure measurements are shown in Fig. 1. It is evident from the data that a change of the barrier height is negligible for the $p$-type AlGaAs. For the $n$-type AlGaAs the pressure coefficients of the barriers are very close to those of the energy gap. In the most recent compilation of these data [11] the pressure coefficient of the direct energy gap is $\mathrm{d} E_{0} / \mathrm{d} p=(10.7-2.7 x) \mathrm{meV} / \mathrm{kbar}$, while for the indirect energy gap $\mathrm{d} E_{\Gamma-X} / \mathrm{d} p=-(1.34+0.3 x) \mathrm{meV} / \mathrm{kbar}$. The measured values (see data in Fig. 1) agree within the experimental error with the band-gap pressure coefficients. We should note that similar trend was seen in a preliminary measurement of the capacitance-voltage $C(V)$ characteristics of the same junctions. Our results are thus consistent with an earlier observation that the pressure shift of the Schottky barrier was equal to the pressure dependence of the band in $n$-type GaAs as derived from $C(V)$ and $I(V)$ measurements $[12,13]$. The results reported here, taken in conjunction with our recent measurements of the influence of temperature on the barrier height, invalidate the midgap theories of the Schottky barrier formation at least in case of almost ideal Al/AlGaAs junctions. 


\section{References}

[1] J. Tersoff, Phys. Rev. Lett. 52, 465 (1984).

[2] F. Flores, C. Tejedor, J. Phys. C 20, 145 (1987).

[3] A. Zunger, Thin Solid Films 104, 301 (1983).

[4] I. Lindau, T. Kendelewicz, CRC Crit. Rev. Solid State Mater. Sci. 13, 27 (1986).

[5] W. Walukiewicz, Appl. Phys. Lett. 54, 2094 (1989) and preceding.

[6] J.M. Langer, Jpn. J. Appl. Phys. 32, 83 (1993).

[7] J.M. Langer, P. Revva, Mater. Sci. Forum 83-87, 1545 (1992).

[8] P. Revva, J.M. Langer, M. Missous, A.R. Peaker, J. Appl. Phys., August (1993).

[9] Ch.G. Van de Walle, Phys. Rev. B 39, 1871 (1989).

[10] M. Missous, W.S. Truscott, K. Singer, J. Appl. Phys. 68, 2239 (1990).

[11] V.A. Wilkinson, A.R. Adams, in: Properties of Aluminium Gallium Arsenide, Ed. S. Adachi, INSPEC, London 1993, p. 82.

[12] W. Shan, M.F. Li, P.Y. Yu, W.L. Hansen, W. Walukiewicz, Appl. Phys. Lett. 53, 974 (1988).

[13] M. Mizuta, B. Toshio, Y. Fujisawa, J. Yoshino, H. Kukimoto, Inst. Phys. Conf. Ser. 106, $321(1990)$. 